\title{
Research on the Passive Electrostatic Detector Technology
}

\author{
Zheng Xing a, Ming Wei, Weidong Liu, Lei Wang and Xinggang Liu \\ Institute of Electrostatic and Electromagnetic Protection, Ordnance Engineering College, \\ Shijiazhuang 050003, China \\ axz1994zheng@163.com
}

\begin{abstract}
Keywords: electrostatic detection; Electrostatic induction; target positioning.
\end{abstract}
\begin{abstract}
The method of locating air flight targets and measuring the speed of air flight targets by the passive electrostatic detectors is studied, and we derive the mathematical expression of the induced current at the detection electrode. The simulation of the change of the induced current with the horizontal distance is carried out, and the simulation results verify the feasibility of detecting the target by detecting the current. Finally, the relationship between the horizontal detection distance and the area of the detection electrode is simulated.
\end{abstract}

\section{Introduction}

Objects with high speed or jet engine will carry static electricity [1]. The static electricity carried by the object forms an electrostatic field that can be detected [2]. Electrostatic detection is a means of detecting the direction, distance, position, speed and other information of the target by detecting the electrostatic field of the electrostatic target. The static electricity generated by the target in flight is difficult to completely eliminate [3], so electrostatic detection has a good anti-stealth ability. Electrostatic detectors can be categorized into two kinds, namely active electrostatic detectors and passive electrostatic detectors. The active electrostatic detector can generate electrostatic field. When the flight target is close to the detector, the detector can capture the target information by detecting the disturbance to the electrostatic field caused by the flying target. The passive electrostatic detector does not generate electrostatic field by itself. When the target is close to the detector, the detector captures the target information by detecting the response of the detector electrode to the electrostatic field generated by the target [4]. Passive electrostatic detectors do not rely on their own launch signal to detect the target, so the passive electrostatic detectors have a good property of self-concealment [5].

\section{Detection principle and detection equation}

The basic principle of electrostatic detection is electrostatic induction. Electrostatic detectors have several kinds of detection electrodes, such as flat-type detection electrode, spherical detection electrode and rotary blade detection electrode [6]. The manufacturing method of the spherical detecting electrode and the rotary blade detecting electrode is complicated, but the flat detecting electrode is simple in manufacture and can be mass-produced.

When the distance between the air flight target and the detection electrode is 6 times greater than the target size, the target can be regarded as a point target [7]. Place the two detection plates in parallel with the ground, and the lower plate is connected with the ground. When the flight target is close to the detection electrode, the electrostatic field produced by the target induces a certain amount of charge on the detection electrode.

The coordinate system is established with the center of the detection electrode as the origin of the coordinates. The coordinate of the target position is $(x, y, z)$. The amount of charge of the target is $+Q$. According from the Coulomb's law, the electric field intensity of the target at the detection electrode [8] is: 


$$
E=\frac{Q}{4 \pi \varepsilon R^{2}}
$$

Wherein $\varepsilon$ is the electrostatic constant and $R$ is the distance between the target and the detection electrode.

The electric field strength perpendicular to the detection electrode is:

$$
E_{n}=E \cdot \sin \theta=\frac{Q}{4 \pi \varepsilon R^{2}} \cdot \frac{z}{R}=\frac{Q z}{4 \pi \varepsilon R^{3}}
$$

$\theta$ in formula (2) is the pitch of the target. The voltage between the two electrodes is:

$$
U=E_{n} \cdot d
$$

$d$ is the vertical distance between two plates. Flat capacitor satisfies:

$$
\begin{aligned}
& -q=C \cdot U \\
& C=\frac{\varepsilon S}{d}
\end{aligned}
$$

Wherein $C$ is the capacitance between the electrodes, $q$ is the induced charge of the electrode, and $S$ is the area of the electrode.

We can derive the amount of induced charge of the detection electrode from formula (2), (3), (4) and (5):

$$
-q=\frac{Q S z}{4 \pi R^{3}}
$$

When the distance between the flight target and the electrode changes, the induced charge on the detection electrode will change. Once the detection electrode and the detection circuit form a loop, the change of charge forms a current. The current [9] is:

$$
i=\frac{d q}{d t}=\frac{d q}{d R} \frac{d R}{d t}=\frac{\partial q}{\partial x} \frac{\partial x}{\partial t}+\frac{\partial q}{\partial y} \frac{\partial y}{\partial t}+\frac{\partial q}{\partial z} \frac{\partial z}{\partial t}
$$

In formula (7), $\frac{\partial x}{\partial t}=v_{x}$ is the velocity of the flight target in the $\mathrm{x}$-axis direction, $\frac{\partial y}{\partial t}=v_{y}$ is the velocity of the flight target in the $\mathrm{y}$-axis direction, $\frac{\partial z}{\partial t}=v_{z}$ is the velocity of the flight target in the $\mathrm{z}$ axis direction, so the induced current can also be expressed as:

$$
i=\frac{Q S}{4 \pi\left(\mathrm{x}^{2}+\mathrm{y}^{2}+\mathrm{z}^{2}\right)^{\frac{5}{2}}}\left[3 z x v_{x}+3 \mathrm{zy} v_{\mathrm{y}}-v_{z}\left(\mathrm{x}^{2}+\mathrm{y}^{2}-2 \mathrm{z}^{2}\right)\right]
$$

From (8) we can see that the induced current is proportional to the amount of charge charged by the target and the area of the detection electrode. And the induced current is related to seven variables, such as target coordinates, charge and speed.

\section{Simulation and Analysis}

For the convenience of analysis, we assume that the target only travels along the y-axis direction, the equation (8) can be simplified as:

$$
i=\frac{3 Q S z \mathrm{y} v_{\mathrm{y}}}{4 \pi\left(\mathrm{x}^{2}+\mathrm{y}^{2}+\mathrm{z}^{2}\right)^{\frac{5}{2}}}
$$

Assume that the target coordinate is $(0, y, 100)$, the target charge is $Q=10^{-15} C$, the area of the electrode is $S=0.25 \mathrm{~m}^{2}$, the target flight speed is $v_{y}=200 \mathrm{~m} / \mathrm{s}$, and the simulation curve is shown in Fig.1. 


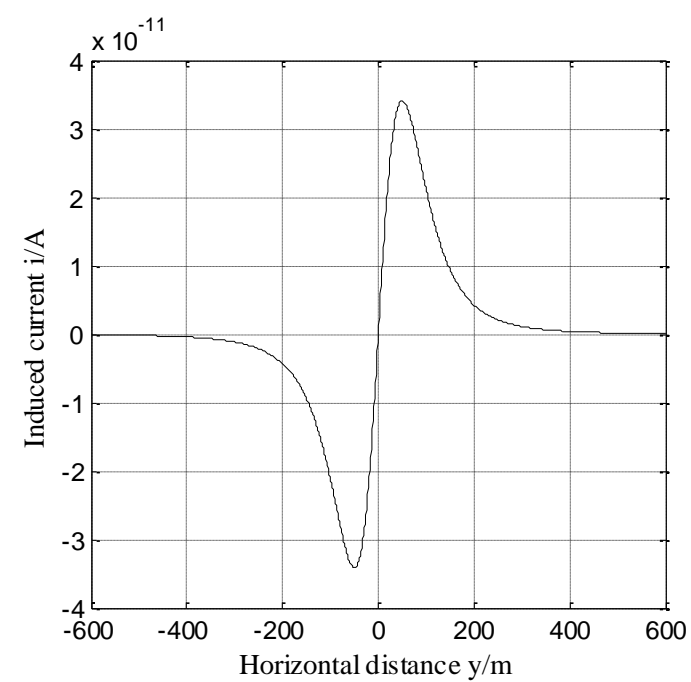

Figure 1. Simulation of the detection equation curve by Matlab software

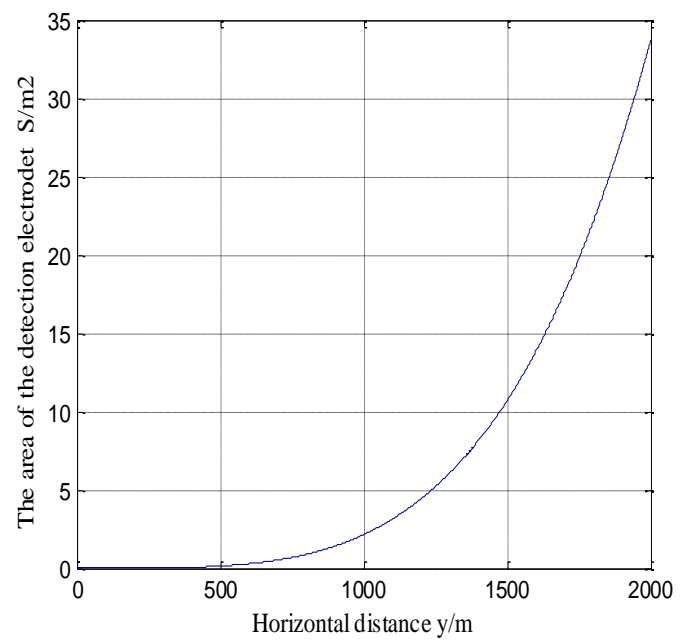

Figure 2. The relationship between the detection horizontal detection distance and electrode area

Femto's LCA series of operational amplifiers gain up to $10^{13} \mathrm{~V} / \mathrm{A}$, the current resolution of $10^{-14} A$ [10]. It can be seen from Fig. 1 that when the vertical distance between target and detection electrode is $100 \mathrm{~m}$ and the horizontal distance is $577 \mathrm{~m}$, the induced current is $10^{-13} \mathrm{~A}$. So, the induced current on the electrostatic detector can be measured. When the distance between the target and the detection electrode becomes smaller, the induction current will increase until the horizontal distance becomes $50 \mathrm{~m}$ where the induced current reaches the peak $\left(3.416 \times 10^{-11} \mathrm{~A}\right)$. Once the horizontal distance is within $50 \mathrm{~m}$, the induced current will decrease. The excitation current is zero when the target is closest to the detection electrode i.e. the target is flying above the detection electrode.

We further study the relationship between the detection distance and the detection electrode area. Assuming that the target charge is $Q=10^{-5} \mathrm{C}$, the induced current is $i=10^{-13} \mathrm{~A}$, the target travels at the speed of $200 \mathrm{~m} / \mathrm{s}$ along the $y$-axis direction, the coordinate is $(0, y, 100)$, the simulation curve is shown in Fig.2.

As can be seen in Fig.2, increasing the detection electrode area can make the detection distance longer, but the effect is very limited. When $y=995 \mathrm{~m}$, the target and the detection electrode distance of $1000 \mathrm{~m}$, then $S=2.102 \mathrm{~m}^{2}$. And it can be achieved by increasing the detection electrode area to achieve the target detection. When $y=1497 \mathrm{~m}$ and the target and the detection electrode distance is $1500 \mathrm{~m}, S$ must satisfy $S=10.64 \mathrm{~m}^{2}$. In this case, the detection electrode area is too large, and it is difficult to produce. The detection of the target can not be achieved by increasing the area of the detector electrode. 


\section{Conclusion}

Based on the basic theory of electrostatic detection, we derive the detection equation of parallel plate electrostatic detector. And we study the relationship of the induced current with the horizontal distance by simulation. And the correctness of the equation is verified. Besides, we study the relationship between the detection distance and the area of the detector electrode. Through simulation, we can get the conclusion that the detection distance can be increased by increasing the detection electrode area within a certain range.

\section{Acknowledgments}

This work was supported by the National Natural Science Foundation of China (No. 51277181).

\section{References}

[1] Sun Keping, Song Guangcheng, Industrial static electricity (China Petrochemical Press, Beijing, 1994), pp. 256-259.

[2] Hill D M, "Time-varying Electrostatic Modeling Techniques," in Report ADA358654, 1999.

[3] Wilkin N D, "The Experimental Determination of the Electric Fields Surrounding a Model Aircraft," in Report ADA043257, 1977.

[4] Aronoff A D, "Electrostatic Means for Intrusion Detection And Ranging," in Report AD-78560, 1965.

[5] Han Lei, Static Detection Mechanism and Application (National Defense Industry Press, Beijing, 2012), pp.2-3.

[6] Chen $\mathrm{Xi}$, Huang Tao, Fu Wei, "Design for electrostatic detection electrode based on finite element analysis," in Transactions of Beijing Institute of Technology, 2011, 31(4), pp. 413-417.

[7] Chen Xi, Cui Zhanzhong, Chen Fang, "Analysis on the Properties of Electrostatic Target," in Transactions of Beijing Institute of Technology, 2005, 25(s), pp. 169-172.

[8] Guo Shuohong, Electrodynamics (Higher Education Press, Beijing, 2008), pp.3-5.

[9] Lin Wei, Cui Zhanzhong, Xu Lixin, et al, "Signal Extraction of Electrostatic Target based on Array Detection," in Journal of Projectiles, Rockets, Missiles and Guidance, 2006, 26(1), pp. 302-304.

[10]Li Peng, "Development of Atmospheric Micro-component Detector," Ph.D. thesis, Chinese Academy of Sciences, 2008, pp. 28-30. 